\title{
Into the Facet-Selectivity of Sequenced Amphiphilic Peptoids at the $\mathrm{Au}$-Water Interface
}

\author{
Xin Qi ${ }^{1}$, Biao Jin' ${ }^{2}$, Bin Cai ${ }^{2,3}$, Feng Yan ${ }^{2,4}$, James De Yoreo ${ }^{1,2}$, Chun-Long Chen ${ }^{1,2}$, Jim \\ Pfaendtner ${ }^{* 1,2}$ \\ ${ }^{1}$ Department of Chemical Engineering, University of Washington, Seattle, 98195 Washington, \\ United States
}

${ }^{2}$ Physical and Computational Sciences Directorate, Pacific Northwest National Laboratory, Richland, Washington 99354, United States

KEYWORDS: Adsorption free-energy, Au, peptoid, solid-liquid interface, selectivity, metadynamics

ABSTRACT: Shape-controlled colloidal nanocrystal syntheses often require aid from facetselective solution-phase chemical additives to regulate atom addition/migration fluxes or oriented particle attachment. Because of their highly tunable chemical property and robustness to a wide range of experimental conditions, peptoids contribute to a very promising group of next-generation functional chemical additives. To generalize the design philosophy, it is critical to understand the origin of facet selectivity at the molecular level. We employ molecular dynamics simulations and biased sampling methods to investigate the origin of $\mathrm{Au}(111)$-favored adsorption of a peptoid, Nce3Ncp6, that is evidenced to assist the formation of five-fold twinned nanostructures. We find 
that the facet-selectivity is achieved through a synergistic effect of both molecule-surface and solvent-surface interactions. Extending beyond the single-chain scenario, the order of peptoidpeptoid and peptoid-surface energetics, i.e., peptoid-Au(100) < peptoid-peptoid < peptoid$\mathrm{Au}(111)$, further amplifies the distinct behavior of Nce3Ncp6 chains on different Au surfaces. Our studies set the stage for future peptoid design in shape-controlled nanocrystal syntheses by probing the facet selectivity from various perspectives. 


\section{Introduction}

In the past two to three decades, the indispensability of solution-phase chemical additives in shape-controlled inorganic nanocrystal syntheses has been repeatedly addressed in experimental literature. ${ }^{1-7}$ Regardless of the chemical composition, these solution-phase additives often, if not always, exhibit facet selectivity and consequently promote the growth of a specific facet or domain.$^{8-14}$ In recent years, with the advancement in computational power and algorithm, theory and simulation works have made numerous breakthroughs in unraveling the complex role of these additives in morphological control.$^{6,15-24}$ For example, with a very high facet selectivity (i.e., when the molecule strongly favors a specific facet over the other), the trend in facet-specific interfacial free energies can be reversed upon adsorption and thus alter the thermodynamic equilibrium shapes. ${ }^{25}$ In the spectrum of weak to moderate facet selectivity, the surface-adsorbed molecules may regulate the solution-phase atom addition fluxes, which gives rise to kinetic shapes. ${ }^{16,17,21,26}$ In addition to the atom-by-atom growth mechanism, facet-selective binding can also guide nanocrystal formation through non-oriented ${ }^{6}$ or oriented attachment ${ }^{27,28}$ by modulating the attachment kinetic barrier, as a result of different packing densities or surface diffusivities of surface adsorbed molecules.

While these chemicals are traditionally selected from polymers, oligomers, proteins, or nucleic acids, peptoids (or $\mathrm{N}$-substituted glycines) contribute to a rising group of candidates as shapedirecting solution-phase chemical additives, ${ }^{6,29,30}$ Being a type of sequence-defined synthetic polymers, peptoids are relatively easy to synthesize and robust to a wide range of experimental conditions. Also, peptoids are chemically similar to peptides, with the side chains connected to the backbone $\mathrm{N}$ atom instead of the alpha $\mathrm{C}$. This makes their properties highly tunable via side chain sequencing. More significantly, the side chain compositions of peptoids are much more diverse 
than naturally occurring amino acids, creating a massive design space for new successful chemical additives with the desired facet-selectivity.

We herein report that a di-block amphiphilic peptoid (Figure 1(a)), Nce3Ncep6, composed of carboxyl $[\mathrm{Nce}=\mathrm{N}-(2$-carboxyethyl)glycines] side chains (hydrophilic) and aromatic $[\mathrm{Ncp}=\mathrm{N}-[2-$ (4-chloro-phenyl)ethyl]glycines)] side chains (hydrophobic) has been observed to mediate the formation of five-fold twinned $\mathrm{Au}$ nanocrystals in a basic aqueous solution $(\mathrm{pH}=9.0)$. More specifically, decahedron-like five-fold twinned nanocrystals are formed with the presence of Nce3Ncep6, while branched or spherical nanocrystals are formed without the peptoid (cf. Figure 1(b) and (c)). Ideally, a single-crystalline nanocrystal of a face-centered cubic (fcc) metal is enclosed by low-index surfaces, such as $\{100\}$ and $\{111\}$ facets, with corners or edges smoothed with $\{110\}$ or higher index facets, to minimize the total surface free energy. Any emergence of twin planes is always associated with energetic costs due to internal strain/stress. ${ }^{31-34}$ One way to counterbalance such energetic penalty is to lower the total surface free energy with surfaceadsorbed chemical additives; otherwise, the structure would undergo reconstruction if the size is small enough (e.g., seeds) or fracture if the structure is too large. ${ }^{8}$ Therefore, a likely route for Nce3Ncep6 to assist the formation of structures in Figure 1(b) is to strongly adsorb to $\mathrm{Au}(111)$, as a decahedron is mostly $\{111\}$-faceted, such that the energy increase associated with the twin plane is sufficiently negatived.

To move forward with structure-directing peptoid design for better shape homogeneity control, it is necessary to establish the linkage between the chemical composition and its influence on majorly involved crystalline facets, such as $\{100\}$ and $\{111\}$ facets. In this work, we use classic molecular dynamics (MD) simulations, together with biased sampling methods, to probe the affinity of Nce3Ncep6 on $\mathrm{Au}(100)$ and $\mathrm{Au}(111)$. We notice a very strong preference of $\mathrm{Au}(111)$ 
over $\mathrm{Au}(100)$. We conclude that it is a synergistic effect from both the peptoid itself and the interfacial water behavior through further analysis. In the end, we move beyond the single-chain properties and shed light on how Nce3Ncep6 may collectively impose influence on the growth of the two facets.

(a)

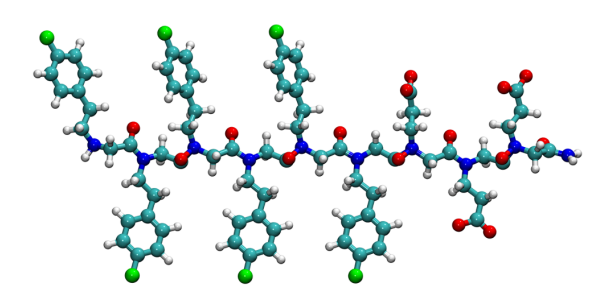

(b)

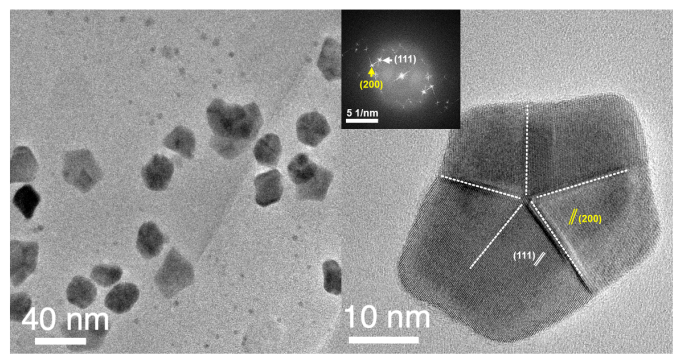

(c)

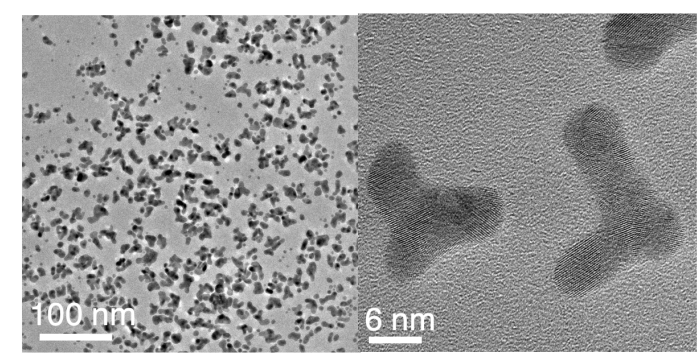

Figure 1. (a) The atomistic structure of Nce3Ncp6 used in MD simulations. The carboxyl groups are deprotonated to reflect the basic synthetic condition. Atoms are colored as the following: $\mathrm{C}$ (cyan), $\mathrm{O}$ (red), $\mathrm{N}$ (blue), $\mathrm{Cl}$ (green), and $\mathrm{H}$ (white). (b) The five-fold twinned nanocrystals synthesized with the presence of Nce3Ncp6 and (c) the branched and small spherical nanocrystals synthesized without the presence of Nce3Ncp6. The twin planes are highlighted with white, dashed lines.

\section{Materials and methods}

All MD simulations are conducted using GROMACS 5.135 with PLUMED 2.4 plugin ${ }^{36}$. Taking the similarity between peptoids and peptides, we describe most of the intra- and intermolecular interactions of Nce3Ncp6 using the CHARMM force field (FF), ${ }^{37,38}$ with few modifications on 
dihedrals to account for the different backbone flexibility. ${ }^{39}$ In addition, we use GolP-CHARMM FF to describe the $\mathrm{Au}-\mathrm{Au}$ and $\mathrm{Au}$-solution interactions for its ability to capture the correct facet selectivity of peptides on Au surfaces in previous studies. ${ }^{40}$ The TIP3P water model is used to be compatible with the GolP-CHARMM parameterization. The initial configuration of each system is designed to accommodate the purpose of the study. Below, we state the configuration setup by category.

\section{Single peptoid on Au surfaces}

We choose metadynamics as the biased sampling method to obtain the adsorption free-energy profiles. To ensure sufficient sampling of the adsorbed and desorbed states of a peptoid near $\mathrm{Au}$, the simulation cell should be large enough to accommodate for exploration in configurational phase space. The $\mathrm{Au}(100)$ system contains a $20 \times 20 \times 8$ (atoms) periodic $\mathrm{Au}(100)$ slab and the $\mathrm{Au}(111)$ system contains a $20 \times 18 \times 8$ (atoms) periodic $\mathrm{Au}(111)$ slab. We insert one peptoid chain near the surface and fill the rest of the simulation box with water, counter ions (Na), and extra ten pairs of $\mathrm{NaCl}$. Both systems are equilibrated in the NPT ensemble using the v-rescale thermostat $(300 \mathrm{~K})$ and Berendsen barostat $(1 \mathrm{bar})$ for $5 \mathrm{~ns},{ }^{41}$ with pressure coupling only applied to the z-dimension (i.e., orthogonal to the surface). Following the simulation scheme recommended by Wright and coworkers, ${ }^{40}$ we freeze the position of Au atoms and virtual sites and only allow the dipolar rods to spin at all times. The final z-dimension is 9.98 and $9.83 \mathrm{~nm}$ for $\mathrm{Au}(100)$ and $\mathrm{Au}(111)$, respectively. Both systems are further equilibrated in the NVT ensemble using the v-rescale thermostat for additional $50 \mathrm{~ns}$ to relax the peptide structure. All production and enhanced sampling runs are carried out in the same NVT ensemble.

The adsorption free-energy of peptoids on $\mathrm{Au}$ surfaces are calculated using parallel-bias metadynamics (PBMetaD) with the multiple-walker scheme.42 In PBMetaD, numerous low 
dimensional collective variables (CV) can be biased in parallel to achieve efficient exploration for high dimensional CVs. The CVs sampled in this study include the distance between the center of mass $(\mathrm{COM})$ of the peptoid backbone and the Au surfaces in z-dimension, the distance between the COM of the side chain of each residue and the Au surfaces in z-dimension, and the radius of gyration $\left(r_{g}\right)$ of the peptoid. The Au surface is represented by the average position of all atoms in the outmost layer. Four walkers are used for each surface, and each walker is run for $1000 \mathrm{~ns}$ to ensure convergence (see the Supporting Information (SI)).

\section{Peptoid-peptoid interaction}

For estimating the energetics between two peptoids, we solvate two Nce3Ncp6 chains in water, counterions, and extra $\mathrm{NaCl}$ ion pairs. The simulation cell dimension is adjusted to $6.04 \mathrm{~nm} \times 6.04 \mathrm{~nm} \times 6.04 \mathrm{~nm}$ after a $5 \mathrm{~ns}$ equilibration in the NPT ensemble with isotropic pressure coupling (v-rescale thermostat and Berendsen barostat), then equilibrated in the NVT ensemble for 50 ns to relax the peptoid structures.

Again, we employ a similar scheme as described above for chain-chain interaction. Similar to single peptoid binding free-energy calculation, four walkers are used, and each walker are sampled for $1000 \mathrm{~ns}$.

\section{Monomer sidechain analogues on Au surfaces}

In the effort to identify the origin of facet selectivity of Nce3Ncp6, we dissect the peptoid into residues and assess their energetic contribution by calculating the adsorption free-energy of side chain analogues. Specifically, we use 1-chloro-4-propyl benzene (Figure 4(a)) and butyrate (Figure 4(f)) for Ncp and Nce analogues, respectively. ${ }^{6}$ A $14 \times 14 \times 8$ (atoms) $A u(100)$ slab and a $15 \times 16 \times 8$ (atoms) $\mathrm{Au}(111)$ slab are used in this study. The equilibration step is carried out in the same set of thermostat and barostat as for the Nce $3 \mathrm{Ncp} 6$ chain, with $5 \mathrm{~ns}$ in the NPT ensemble 
and $20 \mathrm{~ns}$ in the NVT ensemble. Since the side chain analogues lack structure complexity significantly as compared to Nce3Ncp6, we use well-tempered MetaD (WTMetaD) and bias only the orthogonal distance between its COM and the Au surface. The total sampling time is $200 \mathrm{~ns}$ for each analogue and surface combination.

\section{Multiple peptoid chains on Au surfaces}

In addition to single-chain binding on a plain surface, we look into their collective behavior by placing multiple peptoids on the surface. We use the same Au slabs for peptoid adsorption freeenergy sampling here and solvate 30 Nce3Ncp6 peptoids near the surface in water. The relaxed simulation box has a $\mathrm{z}$-dimension of $18.58 \mathrm{~nm}$ and $18.52 \mathrm{~nm}$ for $\mathrm{Au}(100)$ and $\mathrm{Au}(111)$, respectively. The systems are then equilibrated for $800 \mathrm{~ns}$ in the NVT ensemble to arrive at a steady-state structure. We repeat the same process with three more different initial configurations for each surface to ensure the reproducibility of the characteristic configuration.

\section{Results and discussions}

\section{Peptoid-Au and peptoid-peptoid interactions}

We present the free-energy profile of single Nce3Ncp6 chain adsorption on Au surfaces, as well as peptoid dimerization, in Figure 2(a). For peptoid adsorption, the $\mathrm{x}$-axis indicates the distance between the COM of the Nce3Ncp6 chain orthogonal to the surface. Since the COM of the peptoid is not biased during sampling, the free-energy profiles are obtained from the bias through the standard reweighting procedure. To directly compare the preference of a Nce3Ncp6 chain to an Au surface or to another Nce3Ncp6 chain, we superimpose the peptoid dimerization free-energy profiles onto the same plot. For peptoid dimerization, the x-axis indicates the separation of the

COM between two Nce3Ncp6 chains. Since the COM separation between two Nce3Ncp6 chains 
is a spatial vector, we apply a volume correction following Yuan and coworkers, ${ }^{43}$ comparison with and without volume correction can be found in the SI.

A clear message delivered in Figure 2(a) is that Nce3Ncp6 strongly favors $\mathrm{Au}(111)$ over $\operatorname{Au}(100)$. The adsorption free-energy $\Delta F_{a d s}$ can be extracted from the free-energy difference between the adsorbed state $\Delta F_{\text {surf }}$ and the free-floating state in the solution phase $\Delta F_{\text {sol }}$ via $\Delta F_{\text {ads }}=\Delta F_{\text {surf }}-\Delta F_{\text {sol }}{ }^{44-46}$ To calculate $\Delta F_{\text {surf }}$ and $\Delta F_{\text {sol }}$ from Figure 2(a), we draw boundaries (see the SI) to define the adsorbed state and the free-floating state, and obtain the Boltzmann average of free-energy in each region using

$$
\Delta F=-k_{\mathrm{b}} T \log \left[\frac{\int d z e^{-F_{z} / k_{b} T}}{w}\right],
$$

where $k_{b}$ is the Boltzmann constant, $T$ is the temperature, $z$ is the orthogonal distance from the surface, $F_{z}$ is the instant value on the curve at each $z$, and $w$ is the width of the pre-defined region for the state. This approximation yields an affinity of Nce3Ncp6 to $\operatorname{Au}(111)\left(\Delta F_{a d s,\{111\}}=-63.27\right.$ $\mathrm{kJ} / \mathrm{mol})$, nearly eleven-folds higher than that to $\operatorname{Au}(100)\left(\Delta F_{a d s,\{100\}}=-5.44 \mathrm{~kJ} / \mathrm{mol}\right)$, which exhibits a facet selectivity similar to, or even greater than, other successful structure-directing agents for shape-controlled nanocrystal growth. ${ }^{10,47-49}$ 
(a)

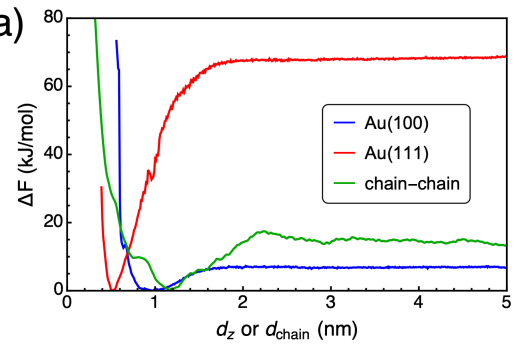

(d)

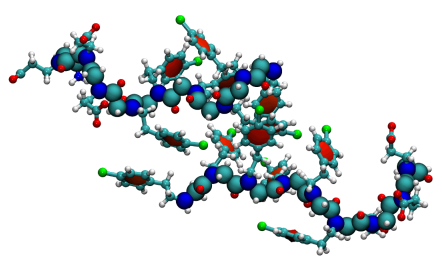

(b)

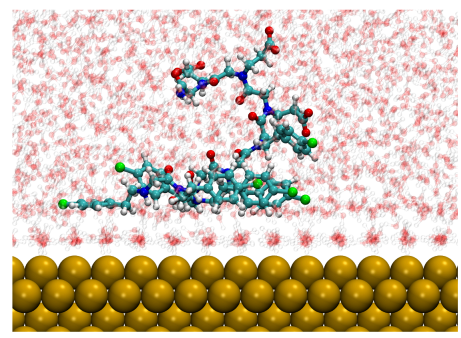

(e)

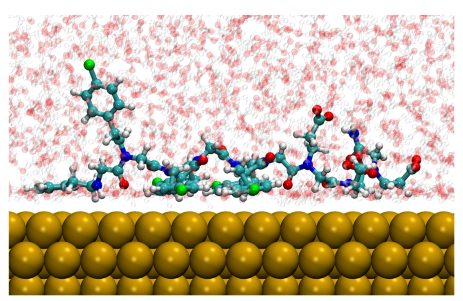

(c)

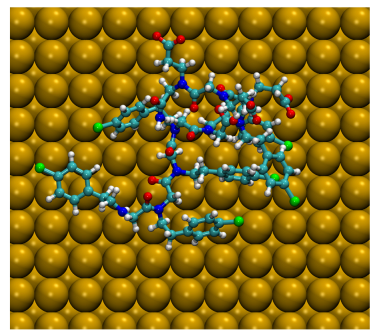

(f)

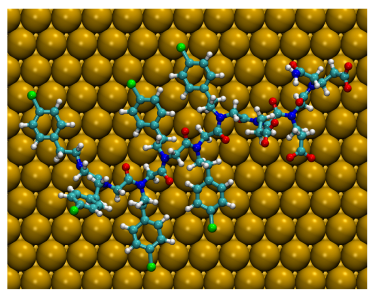

Figure 2. Free-energy profiles and example simulation snapshots. (a) The free-energy profiles of peptoid adsorption on Au facets and peptoid dimerization in water. For surface adsorption, the xaxis indicates the orthogonal distance of the COM of the peptoid relative to the surface, $d_{z}$. For dimerization, the X-axis indicates between the COM of two peptoid chains, $d_{c o m}$. The (b) top and (c) side views of equilibrated Nce3Ncp6 on $\mathrm{Au}(100)$, and the (e) top and (f) side views of equilibrated Nce3Ncp6 on $\mathrm{Au}(111)$. Water molecules are shown in lightened color in the sideview snapshots (oxygen atoms shown in red and hydrogen atoms shown in white) to illustrate their ordered pattern, as well as the relative position of water and peptoid, on Au surfaces. (d) A simulation snapshot of two peptoid chains in water solution. The backbone carbon (cyan) and nitrogen (blue) are shown in large spheres, and chlorobenzene rings on the hydrophobic side chains are filled in red for highlighting purposes.

In addition to the energetic preference, we note that the location of free-energy minimum is further away from $\mathrm{Au}(100)\left(d_{z}=\sim 1.0 \mathrm{~nm}\right)$ than from $\mathrm{Au}(111)\left(d_{z}=\sim 0.5 \mathrm{~nm}\right)$. Atomistic-scale visualization can be helpful to understand the location offset in this case. The side and the top views of an equilibrated Nce3Ncp6 on $\mathrm{Au}(100)$ and $\mathrm{Au}(111)$ are compared in Figure 2. Interestingly, Figure 2(e) shows that the aromatic groups on the hydrophobic side chains can lay flat and close to the $\mathrm{Au}(111)$ facet. In contrast, for $\mathrm{Au}(100)$ in Figure 2(b), a layer of water molecules persists between the peptoid and the surface, regardless of side chain hydrophilicity. We will show the later section that the offset of the free-energy minimum corresponds to the thickness of the stem water layer (i.e., the first peak in Figure 4(a)), and the water molecules in the 
stem layer on $\mathrm{Au}(100)$ are bound tightly to the surface to prohibit general molecular adsorption. Also, in either case, the hydrophilic carboxyl side chains prefer to stay closer to the solution phase rather than the surface.

Another feature in the adsorption free-energy profiles is the relatively narrower well width for $\mathrm{Au}(111)$ than $\mathrm{Au}(100)$, implying the different conformational degrees of freedom in the adsorbed states. By comparing the top and side views in Figure 2, it is evident that, with sufficient backbone and side chain flexibility, the aromatic groups prefer to maximize their contact to $\mathrm{Au}(111)$ by spreading out laterally and positioning the rings parallel to the surface, whereas the peptoid appears more compact on $\mathrm{Au}(100)$. For a more quantitative analysis, we project the $1 \mathrm{D}$ free-energy profile onto an additional $\mathrm{CV}$, the radius of gyration $\left(R_{g}\right)$, to assess the compactness of the protein adsorbed on different facets. By reweighting with respect to both $R_{g}$ and the orthogonal distance from the surface, we obtain 2D free-energy maps as shown in Figure 3.

First, the locations of energy minimum along the y-axis in Figure 3 confirm the difference in compactness as observed in simulation snapshots, that the most energetically preferred $R_{g}$ is smaller on $\mathrm{Au}(100)\left(R_{g,\{100\}}=0.74 \mathrm{~nm}\right)$ than on $\mathrm{Au}(111)\left(R_{g,\{111\}}=0.83 \mathrm{~nm}\right)$. Moreover, by qualitatively comparing the distinct contour shapes on the two maps, we see that the peptoid acts more like compressed 2D blobs on $\mathrm{Au}(111)$ (i.e., more compressed along the $\mathrm{X}$-axis and elongated along the y-axis in Figure 3(b) in contrast to Figure 3(a)), and such confinement stabilizes the molecule on the surface by losing conformation entropy, which lowers the free-energy of the adsorbed state..$^{50}$ 
(a)

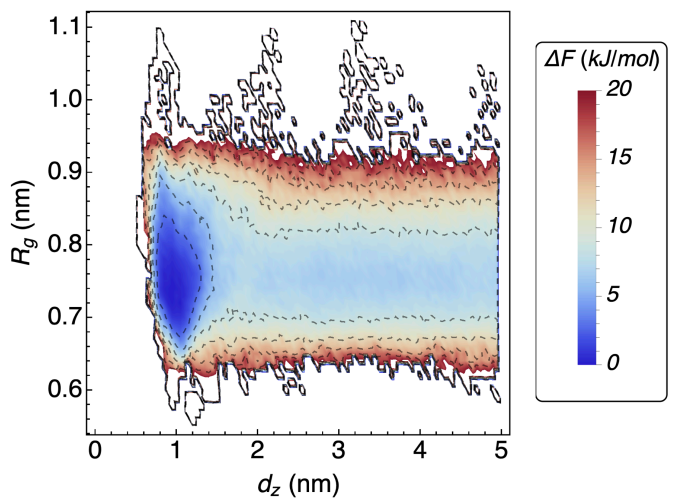

(b)

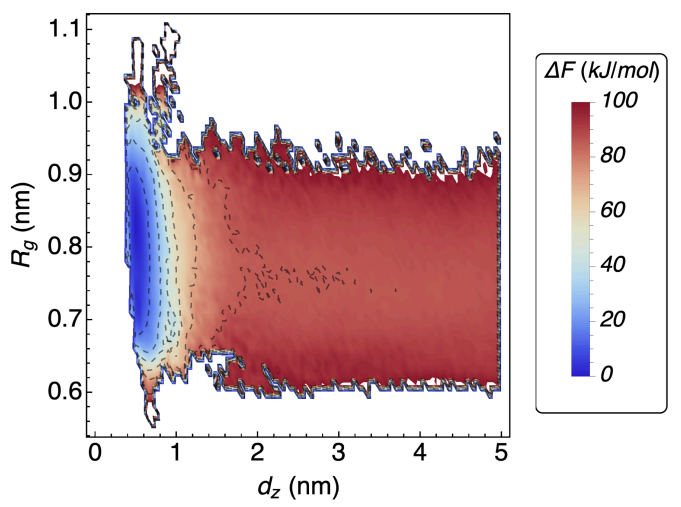

Figure 3. The 2D free-energy map of $R_{g}$ for (a) $\mathrm{Au}(100)$ and (b) $\mathrm{Au}(111)$, respectively. The xaxis indicates the distance between the COM of the peptoid and the surface, and the y-axis indicates the $R_{g}$ of the peptoid. The values of the energy minimum are shifted to 0 for reference. Note that the color scheme corresponds to different energetics scales in (a) and (b).

Aside from the facet selectivity, a direct comparison of peptoid-peptoid interaction to peptoidsurface interaction in Figure 2(d) reveals another interesting character of this facet-selective amphiphilic peptoid, that the peptoid-peptoid affinity is in between its affinity to the two facets, which will be discussed in the last section.

The origin of facet selectivity from the peptoid perspective

The characteristically different interfacial configurations on the two facets shown in the previous section persuasively imply that the energetic preference can be attributed to the interplay among the peptoid-surface, water-surface, and peptoid-water interactions. Herein, we explore the origin of the facet selectivity from both the peptoid and water perspectives. 
As pointed out in the previous section, the Ncp side chains (i.e., the hydrophobic segments) and the Nce side chains (i.e., the hydrophilic segments) exhibit significantly different binding modes on the two facets, as the aromatic groups tend to orient parallel to $\mathrm{Ag}(111)$ and the carboxyl groups are generally further away from both surfaces (c.f. Figure 2 (c), (e), (h), (j)). Thus, our first effort to investigate the origin of facet selectivity is to separate the energetic contribution of each residue type. Here, we assume that the influence of the side chain outweighs that of the backbone, which allows us to model the Ncp and Nce side chains using the analogues as specified in the method section. We obtain their adsorption free-energy on $\mathrm{Au}(100)$ and $\mathrm{Au}(111)$ facets and present the free-energy profiles, as well as the simulation snapshot of equilibrated configurations, in Figure 4.

A comparison of Figures 4 (a) and (f) shows that only the Ncp side chain has a strong preference to $\mathrm{Au}(111)$, whereas the Nce side chain exhibits weak and non-selective binding to either Au facet. It is worth noting that each $\mathrm{Ncp}$ analogue possesses $\sim-10 \mathrm{~kJ} / \mathrm{mol} \Delta F_{\text {ads }}$ difference, which contributes about $1 / 6$ of $\Delta F_{a d s}$ difference for a whole chain. Our observation is consistent with the work of Dasetty, ${ }^{51}$ which also finds that the adsorption free-energy of unstructured peptides on graphene is dominated by the strong binding amino acid residues. The preference of aromatic groups to close-packed coinage metal surfaces via many-body dispersion has been documented in related $a b$ initio studies ${ }^{52,53}$ and is well captured by the GolP-CHARMM FF (cf. Figure 4(b) and (d)). While the location of free-energy minimum for Ncp analogue orthogonal to Au(111) in Figure 4(a) is consistent with a typical equilibrated configuration (Figure 4(e)), we observe two minima for $\mathrm{Au}(100)$, yet the first minimum overlapping with $\mathrm{Au}(111)$ is never observed in unbiased MD simulations. This is because, although the two minima on $\mathrm{Au}(100)$ have the comparable energy level, for a molecule that has arrived at the second minimum from the solution phase, the energy barrier to approach further to the surface is about twice the energy barrier to return to the solution 
phase. Again, based on the width and location of the minima, we confirm that the barrier prohibiting direct surface contact is the stem water layer on $\mathrm{Au}(100)$.

On the contrary, Figure 4(f) to (j) demonstrate that, for neither surface, the interaction between the hydrophilic Nce analogue and the surface is strong enough to break the stem layer network, such that the energetically favored locations for both are above the stem water layer.

Evidence such far confirms the influence of facet selectivity from the molecular perspective of peptoid itself and is embedded with the inseparable role of surface water, which leads to our next step in investigating the facet selectivity.

(a)
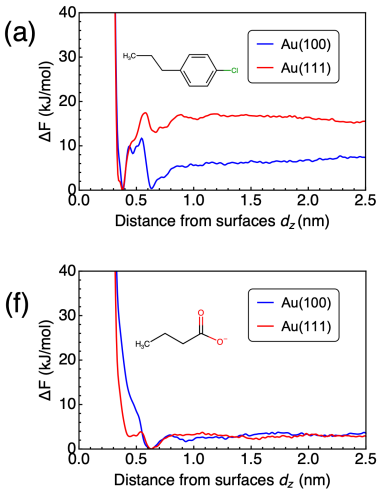

(b)

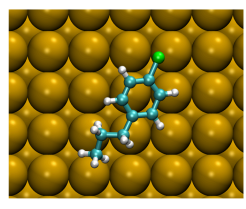

(g)

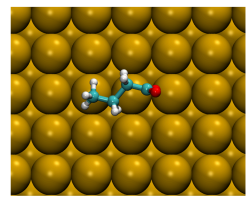

(c)

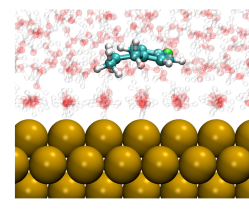

(h)

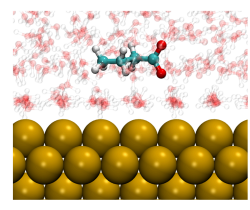

(d)

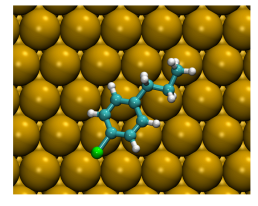

(e)

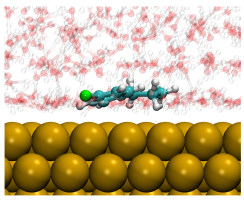

(i)

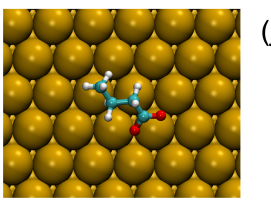

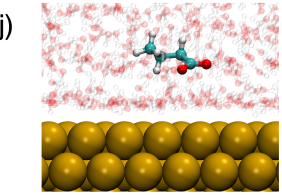

Figure 4. The configurations and adsorption free-energy profiles of side chain analogues. The top and side views of $\mathrm{Ncp}$ analogue on $\operatorname{Au}(100)(\mathrm{a}, \mathrm{b})$ and $\operatorname{Au}(111)(\mathrm{c}, \mathrm{d})$, and the top and side views of Nce analogue on $\mathrm{Au}(100)(\mathrm{f}, \mathrm{g})$ and $\mathrm{Au}(111)(\mathrm{h}, \mathrm{i})$. The adsorption free-energy profile of Ncp and Nce analogues are shown in (e) and (j), respectively. Water molecules are shown in the side views to indicate the relative position of stem layer water and surface-adsorbed molecules.

\section{The origin of facet selectivity from the surface water perspective}

Interfacial water structures and dynamics contrast characteristically to those of the bulk-phase water, and their behavior may depend greatly on the surface chemistry. Previous DFT studies have shown that the $\mathrm{O}$ atoms on water molecules prefer to adopt an a-top configuration on Au. Consequently, due to different packing of $\mathrm{Au}$ atoms in different surface orientations, water 
molecules may exhibit dissimilar orientational or network preferences, consequently affecting the adsorption of other solutes.

Similar to other reported interfaces, water molecules lose translational isotropy and layer up near $\mathrm{Au}$ surfaces. However, the density distribution function of $\mathrm{O}$ atoms in water orthogonal to the surface alone (Figure 5(a)) is too subtle to reconcile the drastic difference we see in previous discussions. On the other hand, we find that water molecules exhibit strikingly dissimilar in-plane features within the stem layer on the two facets (i.e., within the first peak from the surface in Figure 5(a)). To visualize the dissimilarity, we show an ensemble of stem layer water $\mathrm{O}$ atoms positions (labeled as small red dots) relative to the Au atoms underneath (labeled as large golden spheres) in Figures 5(b) and (c). It is clear that nearly all O atoms near the $\mathrm{Au}(100)$ surface align strictly on top of an $\mathrm{Au}$ atom, whereas the distribution of $\mathrm{O}$ atoms is less patterned on $\mathrm{Au}(111)$. To break such ordered water on $\mathrm{Au}(100)$ would require extra free-energy associated with an entropic penalty. In addition, a patterned distribution may also result in a tighter molecular network. We present the distribution of $\theta$, the angle between the water dipole and its projection on the Au surface, of the stem layer water molecules in Figure 5(d). An illustration of how $\theta$ is defined is given in Figure 5(e): a value of $0^{\circ}$ indicates that the dipole is parallel to the surface, and a value of $90^{\circ}$ indicates a dipole aligning perpendicular to the surface, regardless of whether the dipole is pointing up or down. Most noticeably, the two facets differ in the spread of the distributions, where water dipoles near $\mathrm{Au}(100)$ have a preferred angular orientation relative to the surface centered around $45^{\circ}$ but do not exhibit a strongly favored orientation for $\mathrm{Au}(111)$. We also compute the number of hydrogen bonds per water based on the criteria stated in Qi and coworkers. ${ }^{4}$ The number of hydrogen bonds per water molecule in the stem layer is $2.258 \pm 0.006$ for $\mathrm{Au}(100)$ and $2.222 \pm 0.007$ for $\mathrm{Au}(111)$, further evidencing for a tighter and more patterned water network on $\mathrm{Au}(100)$. 
A more ordered molecular network inevitably makes the water molecules more "sticky" to the surface and compete with other adsorptions. To demonstrate the "stickiness," we consider the relaxation dynamics of water in terms of the survival probability $S(t)$, which describes how likely a water molecule will reside in a given layer after a certain time, and also residence time $\tau$ within a 1 ns window (i.e., long relaxation), a characteristic time for survival probability decay. ${ }^{55}$ More stagnant molecular dynamics is often reflected as slow decay in survival probability, i.e., a higher value in $\tau$. The survival probability $S(t)$ is calculated from the ensemble average of $N$ water molecules using

$$
S(t)=\sum_{i=1}^{N}\left\langle\prod_{t_{0}}^{t_{0}+\Delta t} P_{i}^{S}(t)\right\rangle
$$

where $P_{i}^{S}(t)$ is a binary value to find $\left(P_{i}^{S}(t)=1\right)$ or lose $\left(P_{i}^{S}(t)=0\right)$ the $i^{\text {th }}$ water in the first surface layer at time $t$. We plot the normalized $\frac{S(t)}{S(0)}$ in Figure 5(f) for both surfaces, and note that the decay for $\mathrm{Au}(111)$ is much faster than $\mathrm{Au}(100)$, indicating that a surface water molecule on $\mathrm{Au}(111)$ will be more mobile than that on $\mathrm{Au}(100)$. To quantify the decay, we fit the curve to the following equation to find $\tau$

$$
\frac{S(t)}{S(0)}=a_{f} e^{-t / \tau_{f}}+a_{s} e^{-t / \tau_{s}}+c
$$

where $\tau_{f}$ is for the fast component and $\tau_{s}$ is for the slow component in the characteristic decay, and $a_{f}, a_{s}$ and $c$ are coefficients. The fitted value for $\tau_{f}$ is 102 ps for water near $\mathrm{Au}(100)$, and 38 ps for water near $\mathrm{Au}(111)$, and the fitted values for $\tau_{s}$ are $384 \mathrm{ps}$ and $211 \mathrm{ps}$, respectively. Therefore, as a combined result of more ordered static structure, inter-layer network, and slow dynamics, water molecules on $\mathrm{Au}(100)$ form a "protective" layer that effectively impedes the adsorption of any solution-phase molecules. Finally, it should also be pointed out that although the 
stem layer water on $\mathrm{Au}(111)$ is less ordered or stagnant as compared to that on $\mathrm{Au}(100)$, their residency times are still admittedly higher than other surfaces documented in the literature, suggesting a moderately protective water layer. Depending on the interaction between the surface and the incoming solution-phase molecule, such a moderate protective layer may or may not be disrupted, an example of which is the different equilibrium position of Ncp and Nce analogues orthogonal to $\mathrm{Au}(111)$, as shown in Figure 4(d) and (i).

(a)

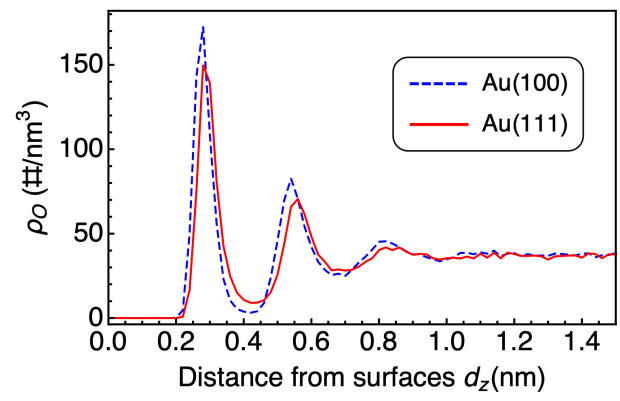

(d)

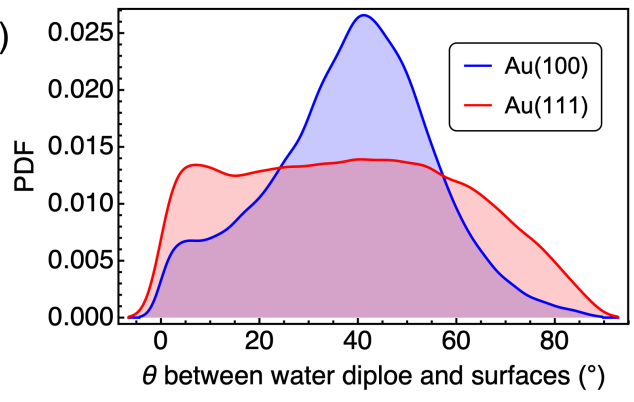

(b)

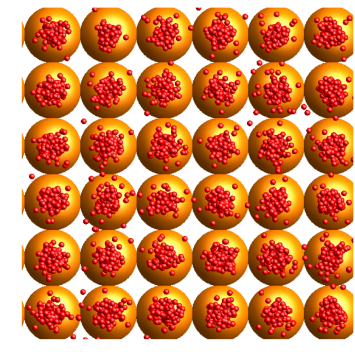

(e)

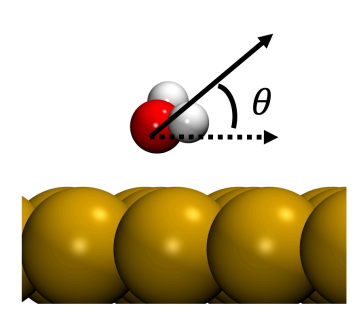

(c)

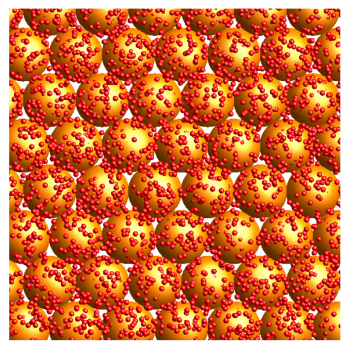

(f)

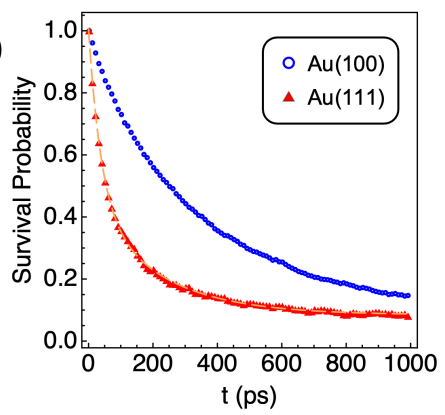

Figure 5. Analyses of the static and dynamic behavior of surface water on $\mathrm{Au}$. (a) The vertical density of $\mathrm{O}$ atoms in the water near the Au surfaces. A collection of the lateral position of $\mathrm{O}$ atoms in the stem layer water (within the first peak in (a)) for (b) $\mathrm{Au}(100)$ and (c) $\mathrm{Au}(111)$, with surface $\mathrm{Au}$ atoms shown in golden spheres underneath for reference. (d) the probability distribution function of the angle between water dipole and surface, $\theta$, for the stem layer water. The definition of $\theta$ is shown in (e). (f) The survival probability of stem layer water over a $1 \mathrm{~ns}$ time window. 


\section{The configurations of the amphiphilic peptoid at a collective level}

Although explaining how the facet selectivity of Nce3Ncp6 is beyond the scope of this paper, we find it is necessary to briefly discuss what ties the cause (i.e., facet selectivity) and the outcome (i.e., nanostructure), that is, the configuration of multiple peptoid chains on Au surfaces. As we have pointed out in the previous section, the free-energy profiles in Figure 2(a) suggest an energetic order of: peptoid-Au(100) < peptoid-peptoid < peptoid-Au(111). An immediate inference we can draw from the trend is that peptoids prefer to maximize their contact with the surface on $\mathrm{Au}(111)$ but with themselves on $\mathrm{Au}(100)$. Together with its amphiphilic nature and that the affinity with the surface is entirely driven by the hydrophobic group, this energetic order results in multiplechain configurations (see Figure S5) that are rarely observed in previous computational studies on other well-known structure-directing agent-mediated nanocrystal synthesis, where relatively simple polymeric or oligomer carpets are evidenced. In Figure S5, peptoids on $\mathrm{Au}(100)$ remain a core-shell cross-sectional structure and sit on top of the stem layer of surface water, whereas peptoids on $\mathrm{Au}(111)$ adopt a bell-like structure, with the aromatic segments (i.e., hydrophobic and favoring $\mathrm{Au}(111))$ buried underneath a cover consisting of the carboxyl groups (i.e., hydrophilic and weakly interacting with $\mathrm{Au}(111))$. These configurations may exert thermodynamics or kinetic influences, or both, on the five-fold nanocrystal formation, for example, greatly passivating $\mathrm{Au}(111)$ by drastically lowing the interfacial free-energy through direct contact or exposing $\mathrm{Au}(100)$ to solution-phase particle or atom addition because the peptoid network is free to diffuse on the surface, etc. A thorough inquiry supported by extensive examination from both the kinetic and thermodynamic aspects is needed to answer these questions.

\section{Conclusions and future work}


To this end, we have shown a very strong selectivity of Nce3Ncp6 to Au(111) over Au(100) using the adsorption free-energy profiles obtained from MD simulation and PBMetaD sampling. Through further analyses on side chain energetic contribution and interfacial water behavior, we identify that the origin of facet selectivity is twofold. From the perspective of the molecular composition of peptoids, the aromatic side chains intrinsically favor the hexagonally packed fcc(111) lattice, and both the backbone dihedrals and the ethyl group on the side chain are flexible enough to maximize the direct contact between the aromatic ring and the surface. Second, water molecules are more ordered and stagnant on $\mathrm{Au}(100)$, which poses a protective barrier to prevent solution-phase molecules from adsorption in general. The carboxyl side chains on Nce3Ncp6, on the other hand, bind weakly and non-selectively to either surface. Due to its hydrophilic nature, we can fairly deduce that an increase in the hydrophilic segment will weaken the peptoid-surface interaction, as the solvation effect will cause the peptoid to be more "lifted" from the surface.

Interestingly, by comparing the peptoid-peptoid and peptoid-surface interactions, we find the energetics appear in the order of peptoid-Au(100) < peptoid-peptoid < peptoid-Au(111). We shed light on how this energetic ordering, together with the amphiphilicity, affects the configuration of peptoids beyond the scope of a single chain. At a collective level, the peptoid configurations, including the fraction of direct surface contact, the molecular diffusivity, and the molecular network, are highly relevant in investigating how the peptoids assist in the nanocrystal formation mechanism and will be the major focus in future studies. 


\section{ASSOCIATED CONTENT}

Additional information on simulation and analysis are stated in detail in the SI.

\section{AUTHOR INFORMATION}

\section{Corresponding Author}

*E-mail: jpfaendt@uw.edu

\section{Notes}

The authors declare no competing financial interest.

\section{ACKNOWLEDGMENT}

This work was funded by the Army Research Office, the Department of Defense, grant number W911NF1920198. This work was facilitated using computational, storage, and networking infrastructure provided by the Hyak supercomputer system, supported in part by the University of Washington and National Science Foundation [CHE-1624430]. Part of this research used the National Energy Research Scientific Computing Center (NERSC) resources, a U.S. Department of Energy Office of Science User Facility located at Lawrence Berkeley National Laboratory, operated under Contract No. DE-AC02-05CH11231.

\section{REFERENCES}

(1) Liu, S.; Guan, Y.; Sheng, Y.; Hu, Y.; Rong, Y.; Mei, A.; Han, H. A Review on Additives for Halide Perovskite Solar Cells. Adv Energy Mater. 2020, 10, 1902492.

(2) Kamat, P. V.; Pradhan, N.; Schanze, K.; Weiss, P. S.; Buriak, J.; Stang, P.; Odom, T. W.; Hartland, G. Challenges and Opportunities in Designing Perovskite Nanocrystal Heterostructures. ACS Energy Lett. 2020, 5, 2253-2255. 
(3) Chan, C.-H.; Poignant, F.; Beuve, M.; Dumont, E.; Loffreda, D. Effect of the Ligand Binding Strength on the Morphology of Functionalized Gold Nanoparticles. J. Phys. Chem. Lett. 2020, 11, 2717-2723.

(4) Fichthorn, K. A Pathway from 3D to 2D. Nat Mater. 2019, 18, 911-912.

(5) Velázquez-Salazar, J. J.; Bazan-Diaz, L.; Zhang, Q.; Mendoza-Cruz, R.; Montaño-Priede, L.; Guisbiers, G.; Large, N.; Link, S.; Jose-Yacaman, M. Controlled Overgrowth of Five-Fold Concave Nanoparticles into Plasmonic Nanostars and Their Single-Particle Scattering Properties. ACS Nano 2019, 13, 10113-10128.

(6) Yan, F.; Liu, L.; Walsh, T. R.; Gong, Y.; El-Khoury, P. Z.; Zhang, Y.; Zhu, Z.; Yoreo, J. J. D.; Engelhard, M. H.; Zhang, X.; Chen, C.-L. Controlled Synthesis of Highly-Branched Plasmonic Gold Nanoparticles through Peptoid Engineering. Nat. Commun. 2018, 9, 1-8.

(7) Xia, Y.; Gilroy, K. D.; Peng, H.-C.; Xia, X. Seed-Mediated Growth of Colloidal Metal Nanocrystals. Angew. Chem. Int. Ed. 2017, 56, 60-95.

(8) Xia, Y.; Xiong, Y.; Lim, B.; Skrabalak, S. E. Shape-Controlled Synthesis of Metal Nanocrystals: Simple Chemistry Meets Complex Physics? Angew. Chem. Int. Ed. 2009, 48, 60103.

(9) Xia, X.; Zeng, J.; Zhang, Q.; Moran, C. H.; Xia, Y. Recent Developments in ShapeControlled Synthesis of Silver Nanocrystals. J. Phys. Chem. C 2012, 116, 21647-21656.

(10) Xia, X.; Zeng, J.; Oetjen, L. K.; Li, Q.; Xia, Y. Quantitative Analysis of the Role Played by Poly(Vinylpyrrolidone) in Seed-Mediated Growth of Ag Nanocrystals. J. Mech. Phys. Solids 2012, 134, 1793-1801.

(11) Silva, R. R. da; Yang, M.; Choi, S.-I.; Chi, M.; Luo, M.; Zhang, C.; Li, Z. Y.; Camargo, P. H. C.; Ribeiro, S. J. L.; Xia, Y. Facile Synthesis of Sub-20 Nm Silver Nanowires through a Bromide-Mediated Polyol Method. ACS Nano 2016, 10, 7892-7900.

(12) Shi, Y.; Lyu, Z.; Zhao, M.; Chen, R.; Nguyen, Q. N.; Xia, Y. Noble-Metal Nanocrystals with Controlled Shapes for Catalytic and Electrocatalytic Applications. Chem. Rev. 2020, 121, 649-735.

(13) Yang, T.; Shi, Y.; Janssen, A.; Xia, Y. Surface Capping Agents and Their Roles in ShapeControlled Synthesis of Colloidal Metal Nanocrystals. Angew. Chem. Int. Ed. 2020, 59, 1537815401.

(14) Ruan, L.; Chiu, C.-Y.; Li, Y.; Huang, Y. Synthesis of Platinum Single-Twinned Right Bipyramid and $\{111\}$-Bipyramid through Targeted Control over Both Nucleation and Growth Using Specific Peptides. Nano Lett. 2011, 11, 3040-3046. 
(15) Chen, Z.; Fichthorn, K. A. Adsorption of Ethylenediamine on Cu Surfaces: Attributes of a Successful Capping Molecule Using First-Principles Calculations. Nanoscale 2021, 13, 13529_ 13537.

(16) Qi, X.; Balankura, T.; Zhou, Y.; Fichthorn, K. A. How Structure-Directing Agents Control Nanocrystal Shape: Polyvinylpyrrolidone-Mediated Growth of Ag Nanocubes. Nano Lett. 2015, $15,7711-7717$.

(17) Kim, M. J.; Alvarez, S.; Yan, T.; Tadepalli, V.; Fichthorn, K. A.; Wiley, B. J. Modulating the Growth Rate, Aspect Ratio, and Yield of Copper Nanowires with Alkylamines. Chem Mater. 2018, 30, 2809-2818.

(18) Hughes, Z. E.; Wright, L. B.; Walsh, T. R. Biomolecular Adsorption at Aqueous Silver Interfaces: First-Principles Calculations, Polarizable Force-Field Simulations, and Comparisons with Gold. Langmuir 2013, 29, 13217-13229.

(19) Balankura, T.; Qi, X.; Zhou, Y.; Fichthorn, K. A. Predicting Kinetic Nanocrystal Shapes through Multi-Scale Theory and Simulation: Polyvinylpyrrolidone-Mediated Growth of Ag Nanocrystals. J. Chem. Phys. 2016, 145, 144106.

(20) Heinz, H.; Pramanik, C.; Heinz, O.; Ding, Y.; Mishra, R. K.; Marchon, D.; Flatt, R. J.; Estrela-Lopis, I.; Llop, J.; Moya, S.; Ziolo, R. F. Nanoparticle Decoration with Surfactants: Molecular Interactions, Assembly, and Applications. Surf. Sci. Rep. 2017, 72, 1-58.

(21) Kim, M. J.; Alvarez, S.; Chen, Z.; Fichthorn, K. A.; Wiley, B. J. Single-Crystal Electrochemistry Reveals Why Metal Nanowires Grow. J. Am. Chem. Soc. 2018, 140 (44), $14740-14746$.

(22) Fichthorn, K. A.; Chen, Z. Surface Science of Shape-Selective Metal Nanocrystal Synthesis from First-Principles: Growth of $\mathrm{Cu}$ Nanowires and Nanocubes. J Vac. Sci. Technol. A 2020, 38, 023210.

(23) Tang, Z.; Zhang, Q.; Yin, Y.; Chang, C. A. Facet Selectivity of Ligands on Silver Nanoplates: Molecular Mechanics Study. J. Phys. Chem. C 2014, 118, 21589-21598.

(24) Murshid, N.; Kitaev, V. Role of Poly(Vinylpyrrolidone) (PVP) and Other Sterically Protecting Polymers in Selective Stabilization of $\{111\}$ and $\{100\}$ Facets in Pentagonally Twinned Silver Nanoparticles. Chem. Commun. 2014, 50, 1247-1249.

(25) Qi, X.; Fichthorn, K. A. Theory of the Thermodynamic Influence of Solution-Phase Additives in Shape-Controlled Nanocrystal Synthesis. Nanoscale 2017, 9, 15635-15642.

(26) Salvalaglio, M.; Vetter, T.; Mazzotti, M.; Parrinello, M. Controlling and Predicting Crystal Shapes: The Case of Urea. Angew. Chem. Int. Ed. 2013, 52, 13369-13372. 
(27) Balankura, T.; Yan, T.; Jahanmahin, O.; Narukatpichai, J.; Ng, A.; Fichthorn, K. A. Oriented Attachment Mechanism of Triangular Ag Nanoplates: A Molecular Dynamics Study. Nanoscale Adv 2020, 2, 2265-2270.

(28) Liu, C.; Chen, Q. Interfacial Crystallization under DNA Control. Nat Mater. 2020, 19, 704705.

(29) Yang, W.; Yin, Q.; Chen, C.-L. Designing Sequence-Defined Peptoids for Biomimetic Control over Inorganic Crystallization. Chem. Mater. 2021, 33, 3047-3065.

(30) Li, R.; Smolyakova, A.; Maayan, G.; Rimer, J. D. Designed Peptoids as Tunable Modifiers of Zeolite Crystallization. Chem. Mater. 2017, 29, 9536-9546.

(31) Cleveland, C. L.; Landman, U. The Energetics and Structure of Nickel Clusters: Size Dependence. J. Chem. Phys. 1991, 94, 7376-7396.

(32) Casillas, G.; Velázquez-Salazar, J. J.; Jose-Yacaman, M. A New Mechanism of Stabilization of Large Decahedral Nanoparticles. J. Phys. Chem. C 2012, 116, 8844-8848.

(33) Patala, S.; Marks, L. D.; Cruz, M. O. de la. Elastic Strain Energy Effects in Faceted Decahedral Nanoparticles. J. Phys. Chem. C 2013, 117, 1485-1494.

(34) Patala, S.; Marks, L. D.; Cruz, M. O. de la. Thermodynamic Analysis of Multiply Twinned Particles: Surface Stress Effects. J. Phys. Chem. Lett. 2013, 4, 3089-3094.

(35) Abraham, M. J.; Murtola, T.; Schulz, R.; Páll, S.; Smith, J. C.; Hess, B.; Lindahl, E. GROMACS: High Performance Molecular Simulations through Multi-Level Parallelism from Laptops to Supercomputers. Softwarex 2015, 1, 19-25.

(36) Tribello, G. A.; Bonomi, M.; Branduardi, D.; Camilloni, C.; Bussi, G. PLUMED 2: New Feathers for an Old Bird. Comput. Phys. Commun. 2014, 185, 604-613.

(37) Vanommeslaeghe, K.; Hatcher, E.; Acharya, C.; Kundu, S.; Zhong, S.; Shim, J.; Darian, E.; Guvench, O.; Lopes, P.; Vorobyov, I.; MacKerell, A. D. J. CHARMM General Force Field: A Force Field for Drug-Like Molecules Compatible with the CHARMM All-Atom Additive Biological Force Fields. J. Comput. Chem. 2010, 31, 671-690.

(38) Huang, J.; MacKerell, A. D. CHARMM36 All-atom Additive Protein Force Field: Validation Based on Comparison to NMR Data. J Comput. Chem. 2013, 34, 2135-2145.

(39) Mirijanian, D. T.; Mannige, R. V.; Zuckermann, R. N.; Whitelam, S. Development and Use of an Atomistic CHARMM-based Forcefield for Peptoid Simulation. J Comput. Chem 2014, 35 (5), 360-370. 
(40) Wright, L. B.; Rodger, P. M.; Corni, S.; Walsh, T. R. GolP-CHARMM: First-Principles Based Force Fields for the Interaction of Proteins with $\mathrm{Au}(111)$ and $\mathrm{Au}(100)$. J. Chem. Theory Comput. 2013, 9, 1616-1630.

(41) Berendsen, H. J. C.; Postma, J. P. M.; Gunsteren, W. F. V.; DiNola, A.; Haak, J. R. Molecular Dynamics with Coupling to an External Bath. J. Chem. Phys. 1984, 81, 3684-3690.

(42) Pfaendtner, J.; Bonomi, M. Efficient Sampling of High-Dimensional Free-Energy Landscapes with Parallel Bias Metadynamics. J. Chem. Theory Comput. 2015, 11, 5062-5067.

(43) Yuan, F.; Wang, S.; Larson, R. G. Potentials of Mean Force and Escape Times of Surfactants from Micelles and Hydrophobic Surfaces Using Molecular Dynamics Simulations. Langmuir 2015, 31, 1336-1343.

(44) Mao, C. M.; Sampath, J.; Sprenger, K. G.; Drobny, G.; Pfaendtner, J. Molecular Driving Forces in Peptide Adsorption to Metal Oxide Surfaces. Langmuir 2019, 35, 5911-5920.

(45) Sampath, J.; Pfaendtner, J. Amphiphilic Peptide Binding on Crystalline vs. Amorphous Silica from Molecular Dynamics Simulations. Mol. Phys. 2019, 1, 1-9.

(46) Sultan, A. M.; Westcott, Z. C.; Hughes, Z. E.; Palafox-Hernandez, J. P.; Giesa, T.; Puddu, V.; Buehler, M. J.; Perry, C. C.; Walsh, T. R. Aqueous Peptide-TiO2 Interfaces: Isoenergetic Binding via Either Entropically or Enthalpically Driven Mechanisms. ACS Appl. Mater. Interfaces 2016, 8, 18620-18630.

(47) Balankura, T.; Qi, X.; Fichthorn, K. A. Solvent Effects on Molecular Adsorption on Ag Surfaces: Polyvinylpyrrolidone Oligomers. J. Phys. Chem. C 2018, 122, 14566-14573.

(48) Liu, S.-H.; Fichthorn, K. A. Interaction of Alkylamines with Cu Surfaces: A Metal-Organic Many-Body Force Field. J. Phys. Chem. C 2017, 121, 22531-22541.

(49) Ruan, L.; Ramezani-Dakhel, H.; Chiu, C.-Y.; Zhu, E.; Li, Y.; Heinz, H.; Huang, Y. Tailoring Molecular Specificity Toward a Crystal Facet: A Lesson From Biorecognition Toward Pt $\{111\}$. Nano Lett. 2013, 13, 840-846.

(50) Rubinstein, M. and Colby, R. Polymer Physics, 1st ed.; Oxford University Press, 003.

(51) Dasetty, S.; Sarupria, S. Advancing Rational Control of Peptide-Surface Complexes. $J$ Phys. Chem. B 2021, 125, 2644-2657.

(52) Jiang, Y.; Yang, S.; Li, S.; Liu, W. Aromatic Molecules on Low-Index Coinage Metal Surfaces: Many-Body Dispersion Effects. Sci. Rep. 2016, 6, 1-8.

(53) Liu, W.; Carrasco, J.; Santra, B.; Michaelides, A.; Scheffler, M.; Tkatchenko, A. Benzene Adsorbed on Metals: Concerted Effect of Covalency and van Der Waals Bonding. Phys. Rev. B $2012,86,245405$. 
(54) Qi, X.; Zhou, Y.; Fichthorn, K. A. Obtaining the Solid-Liquid Interfacial Free Energy via Multi-Scheme Thermodynamic Integration: Ag-Ethylene Glycol Interfaces. J. Chem. Phys. 2016, $145,194108$.

(55) Debnath, A.; Mukherjee, B.; Ayappa, K. G.; Maiti, P. K.; Lin, S.-T. Entropy and Dynamics of Water in Hydration Layers of a Bilayer. J. Chem. Phys. 2010, 133, 174704. 